\title{
The influence of the alpha-adducin G460W polymorphism and angiotensinogen M235T polymorphism on antihypertensive medication and blood pressure
}

\author{
Hedi Schelleman ${ }^{1,2}$, Olaf H Klungel ${ }^{2}$, Jacqueline CM Witteman ${ }^{1}$, Albert Hofman ${ }^{1}$, \\ Cornelia M van Duijn ${ }^{1}$, Anthonius de Boer ${ }^{2}$, Bruno HCH Stricker ${ }^{*}$
}

\footnotetext{
${ }^{1}$ Department of Epidemiology and Biostatistics, Erasmus MC, Rotterdam, The Netherlands; ${ }^{2}$ Department of Pharmacoepidemiology and Pharmacotherapy, Utrecht Institute for Pharmaceutical Sciences (UIPS), Utrecht University, Utrecht, The Netherlands
}

Despite the availability of a variety of effective antihypertensive drugs, inadequate control of blood pressure is common in hypertensive patients. The aim of this study was investigate whether the $\alpha$-adducin G460W polymorphism or angiotensinogen M235T polymorphism has an effect on the mean difference in blood pressure in subjects using antihypertensive drugs. Data from the Rotterdam Study, a populationbased prospective cohort study in the Netherlands, was used. This study started in 1990 and included 7983 subjects of $\mathbf{5 5}$ years and older. Data from three examination rounds were used. Subjects were included when their blood pressure was elevated at 1 or more examinations and/or a diuretic, $\beta$-blocker, calcium antagonist, or ACE inhibitor was used. A marginal generalized linear model was used to assess the druggene interaction. In total, 3025 hypertensives were included. No drug-gene interaction on blood pressure levels was found. The mean difference in systolic blood pressure (SBP) between subjects with the W-allele and GG genotype of the $\alpha$-adducin gene was for diuretic users $1.25 \mathrm{mmHg}(95 \% \mathrm{Cl}:-2.86$ to 5.35$)$, for $\beta$-blockers $0.02 \mathrm{mmHg}(95 \% \mathrm{Cl}:-3.39$ to 3.42$)$, for calcium antagonists $-0.70 \mathrm{mmHg}$ (95\% Cl: -5.61 to 4.21), and for ACE inhibitors $-3.50 \mathrm{mmHg}(95 \% \mathrm{Cl}:-9.02$ to 2.02). The mean difference in SBP between subjects with the TT and MM genotype was for diuretic users $-2.33 \mathrm{mmHg}$ (95\% CI:-8.32 to 3.66), for $\beta$-blocker $-0.06 \mathrm{mmHg}(95 \% \mathrm{Cl}:-4.91$ to 4.79$)$, for calcium antagonist $0.59 \mathrm{mmHg}(95 \% \mathrm{Cl}-5.95$ to 7.13$)$, and for ACE inhibitor $-2.33 \mathrm{mmHg}(95 \% \mathrm{Cl}:-9.66$ to 5.01$)$. The G460W polymorphism and the M235T polymorphism did not modify the difference in blood pressure levels among subjects who used diuretics, $\beta$-blockers, calcium antagonists, or ACE inhibitors.

European Journal of Human Genetics (2006) 14, 860-866. doi:10.1038/sj.ejhg.5201632; published online 17 May 2006

Keywords: alpha-adducin; angiotensinogen; drug-gene interaction; blood pressure; antihypertensive drugs

\footnotetext{
*Correspondence: Professor BHCH Stricker, Department of Epidemiology and Biostatistics, Erasmus MC, PO Box 1738, 3000 DR Rotterdam, The Netherlands;

Tel: + 3110 4087482; Fax: + 3110 4089382;

E-mail: b.stricker@erasmusmc.nl

Received 21 July 2005; revised 5 January 2006; accepted 14 March 2006; published online 17 May 2006
}

Introduction

Hypertension is the most prevalent cardiovascular risk factor in the industrialized world. Despite the availability of a variety of effective antihypertensive drugs, inadequate control of blood pressure is common in hypertensive patients. This is caused by environmental and genetic factors. 
A number of studies have investigated genetic polymorphisms as determinants of cardiovascular response to antihypertensive drug therapy, for example, the G460W polymorphism of the $\alpha$-adducin (ADD1) gene and the M235T polymorphism of the angiotensinogen (AGT) gene. In five studies, the drug-gene interaction was evaluated between the G460W polymorphism of the ADD1 gene and antihypertensive drugs on blood pressure response ${ }^{1-5}$ (see Table 1). Three studies, with partly the same study population, found a greater blood pressure reduction with the $460 \mathrm{~W}$ allele than with the $460 \mathrm{G}$ allele, ${ }^{1-3}$ while the other two studies could not replicate this finding. ${ }^{4,5}$ All these studies were nonrandomized trials. In an observational study, the $460 \mathrm{~W}$ allele was associated with a lower risk of MI or stroke in users of diuretics in comparison to other antihypertensive drug therapy. ${ }^{6}$ Three research groups studied the influence of the M235T polymorphism of the AGT gene on blood pressure response to antihypertensive medication. ${ }^{7-9}$ Two of these studies where nonrandomized trials ${ }^{7,9}$ and the other study was a placebocontrolled crossover trial. ${ }^{8}$ Hypertensive subjects with the $235 \mathrm{~T}$ allele when treated with ACE inhibitors had a greater blood pressure reduction in one study, ${ }^{7}$ but this could not be reproduced in another study. ${ }^{8}$ No drug-gene interactions were found with $\beta$-blockers or calcium antagonists. ${ }^{8,9}$ No interaction was found between the M235T polymorphism and the use of ACE-inhibitors on wall thickness. ${ }^{10}$ Bis et al reported that the $235 \mathrm{~T}$ allele was associated with a lower risk of stroke in ACE-inhibitor users, although this was not found for the risk of myocardial infarction. ${ }^{11}$

The purpose of this study was to evaluate the relationship between the G460W polymorphism of the $\alpha$-adducin gene and the M235T polymorphism of the angiotensinogen gene on the mean difference in blood pressure levels in hypertensive patients when treated with diuretics, $\beta$-blockers, calcium antagonists, and ACE inhibitors in daily practice.

\section{Methods \\ Setting}

The Rotterdam Study started in 1990 as a population-based prospective follow-up study. All 10275 residents of the suburb Ommoord in Rotterdam, aged 55 years or older were invited to participate. In total, 7983 (78\%) subjects gave written informed consent and of $86 \%$ of them blood samples were available for genotyping. The baseline measurements took place until 1993 and the design has been described elsewhere. ${ }^{12}$ The baseline examination included several details, such as an interview on demographics, current health status, medical history, family history of diseases, smoking habits, and current use of medication. During a physical examination, blood pressure, weight, and height were measured, and blood was drawn for DNA extraction. Blood pressure was measured in sitting position at the right upper arm with a random-zero sphygmomanometer. The average of the two measurements, separated by a count of pulse rate, was used in the analysis. All participants were subsequently examined in follow-up examination rounds every 2-3 years (19931995, 1997-1999). Blood pressure from all three examinations were used in this study.

Pharmacy records were available for approximately $99 \%$ of the cohort as of January, 1 1991. These records include

Table 1 Influence of the ADD1 G460W and AGT M235T polymorphism on the effect of antihypertensive therapy in patients with essential hypertension

\begin{tabular}{|c|c|c|c|c|}
\hline Sample & $\begin{array}{l}\text { Duration of } \\
\text { therapy }\end{array}$ & Gene+polymorphism & Allelic association & Reference \\
\hline \multicolumn{5}{|l|}{ Diuretic } \\
\hline$N=143$ Caucasian & 8 weeks & ADD1 G460W & 460W allele associated with greater BP response & $1-3$ \\
\hline$N=585$ Mixed & 2 months & ADD1 G460W & No association (BP) & 4 \\
\hline$N=76$ Asian & 2 months & ADD1 G460W & No association (BP) & 5 \\
\hline$N=1038$ Mixed & 4 years & ADD1 G460W & $\begin{array}{l}460 \mathrm{~W} \text { allele associated with a lower risk of } \mathrm{Ml} \text { or } \\
\text { stroke in comparison to other antihypertensive } \\
\text { therapies (observational study) }\end{array}$ & \\
\hline \multicolumn{5}{|l|}{ ACE inhibitor } \\
\hline$N=125$ Caucasian & 4 weeks & AGT M235T & 235T allele associated with greater BP response & 7 \\
\hline$N=63-91$ Caucasian & 4 weeks & AGT M235T & No association (BP) & 8 \\
\hline$N=56$ Caucasian & 5 months & AGT M235T & No association (wall thickness) & 10 \\
\hline$N=1241$ Mixed & 4 years & AGT M235T & $\begin{array}{l}235 \mathrm{~T} \text { allele associated with a lower risk of stroke in } \\
\text { comparison to nonuse (no association MI) } \\
\text { (observational study) }\end{array}$ & 11 \\
\hline \multicolumn{5}{|l|}{ Beta blockers } \\
\hline$N=63-91$ Caucasian & 4 weeks & AGT M235T & No association (BP) & 8 \\
\hline$N=86$ Caucasian & 3 months & AGT M235T & No association (BP' and LVM) & 9 \\
\hline
\end{tabular}

$\mathrm{ADD1}=$ alpha-adducin; $\mathrm{AGT}=$ angiotensinogen; $\mathrm{BP}=$ blood pressure; $\mathrm{LVM}=$ left ventricular mass. 
the name of the drug, the day of dispensing, the dosage form, the number of units dispensed, the prescribed daily dose, and the Anatomical Therapeutic Chemical code of the drug. ${ }^{13}$

\section{Cohort and outcome definition}

The study population included all individuals in the Rotterdam Study for whom the ACE genotype was assessed. In addition, during follow-up individuals had to have hypertension. Hypertension was defined as $\geqslant 1$ measurement with a systolic blood pressure (SBP) above $160 \mathrm{mmHg}$ and/or diastolic blood pressure (DBP) above $95 \mathrm{mmHg}$, and/or use of one antihypertensive drug at the time of a blood pressure measurement (monotherapy). When a blood pressure measurement occurred, we assessed whether a prescription was filled by the pharmacy on this date. Hereto, the length of each prescription was calculated by dividing the number of dispensed tablets or capsules by the prescribed daily number. When the blood pressure measurement date fell within the usage period, the patient was considered currently exposed. Blood pressure measurements were excluded when combinations of antihypertensive drugs were used. Subjects could switch between no treatment and different antihypertensive drug classes. Only subjects using diuretics, $\beta$-blockers, calcium antagonists, and ACE inhibitors were included in the analysis, because of the small numbers for the other antihypertensive drug classes. Pharmacy records were available as of January 1, 1991. Nevertheless, blood pressure measurements taken in 1990 were included if an individual did not use an antihypertensive drug according to self-reported questionnaire information and did not start antihypertensive therapy before July 1, 1991. The end of the study period was set at December 31, 1999.

In addition, we distinguished between starters and continuous users. Starters were defined as hypertensives who did not have a prescription before July 1, 1991 and used their antihypertensive medication, which was used during the blood pressure measurement, less then 6 of the 8 weeks prior to their blood pressure measurement. In addition, there had to be at least a 30 days gap between the start date of prescription used during the blood pressure measurement and the end date of the previous prescription.

\section{Potential confounders}

As potential confounders, we considered age, sex, body mass index, defined daily dose, (re-)examination round (1st, 2nd, 3rd), smoking at baseline, salt intake at baseline (g/day), history of myocardial infarction, diabetes mellitus at baseline, use of nitrates, use of statins, use of NSAIDs, the use of another antihypertensive drug class 2 weeks prior to the blood pressure measurement, the use of an antihypertensive drug 6 of the 8 weeks prior to the blood pressure measurement, and the cumulative number of days an antihypertensive drug was used.

History of myocardial infarction was self-reported confirmed by a physician or demonstrated on the baseline ECG. To compare dosages of different antihypertensive drugs in our analysis, we used the prescribed daily dose, expressed as the number of DDDs (defined daily doses) per day. The DDD is defined as the recommended dose for the main indication in an adult of $70 \mathrm{~kg} .{ }^{13}$

\section{Genotype}

Genomic DNA was extracted from whole-blood samples using standard methods, described previously. ${ }^{14}$ Samples were genotyped with TaqMan allelic discrimination AssaysBy-Design (Applied Biosystems, Foster City, CA, USA). Forward and reverse primer sequences were $5^{\prime}$-GAG AAG ACA AGA TGG CTG AAC TCT- $3^{\prime}$ and $5^{\prime}$-GTC TTC GAC TTG GGA CTG CTT-3' and the minor groove binding probes were 5'-ATT CTG CCA TTC CTC-3' (VIC) and 5'-ATT CTG CCA TTC CTC-3' (FAM) for the ADD1 gene. Forward and reverse primer (antisense strand) sequences were $5^{\prime}$-AGG TTT GCC TTA CCT TGG AAG TG-3' and 5'-GCT GTG ACA GGA TGG AAG ACT-3' and the minor groove binding probes were 5'-CTG GCT CCC ATC AGG-3' (VIC) and 5'CTG GCT CCC GTC AGG-3' (FAM) for the AGT gene. The assays utilized $5 \mathrm{ng}$ of genomic DNA and $2 \mathrm{ml}$ reaction volumes. The amplification and extension protocol was as follows: an initial activation step of $10 \mathrm{~min}$ at $95^{\circ} \mathrm{C}$ preceded 40 cycles of denaturation at $95^{\circ} \mathrm{C}$ for $15 \mathrm{~s}$ and annealing and extension at $50^{\circ} \mathrm{C}$ for $60 \mathrm{~s}$. Allele-specific fluorescence was then analyzed on an ABI Prism 7900HT Sequence Detection System with SDS v 2.1 (Applied Biosystems, Foster City, CA, USA).

\section{Analysis}

We used ANOVA (continuous variables) and $\chi^{2}$ testing (categorical variables) to compare baseline characteristics of people with different genotypes. To compare the difference in DDD in each examination between the genotype groups ANOVA was used, stratified for the different genotypes. A marginal generalized linear model (GEE) was used to study the potential interaction between the $\alpha$-adducin G460W, angiotensinogen M235T polymorphism, and response to antihypertensive treatment for two outcomes: mean difference in SBP and in DBP. Since subjects could have 1, 2, or 3 blood pressure measurements, the GEE model was used to account for intraperson correlations between repeated measurements. The covariance matrix of the repeated dependent measurements was unstructured and data were analysed using SAS statistical software and adjusted for potential confounders (SAS version 8.2).

We performed two separate analysis. In the first analysis, we compared the mean SBP and DBP measurements between the different genotype groups between users of 
antihypertensive drug class in question (drug-gene interaction). To test for the interaction between the polymorphism in question (eg ADD1 G460W) and the use of the antihypertensive drug class in question (eg low-ceiling diuretics), one dummy was added in the model: ADD1 genotype $(\mathrm{GW}$ or $\mathrm{WW}) \times$ the use of a low-ceiling diuretic during the blood pressure measurement. For the AGT M235T polymorphism, two dummies were added to the model, that is, AGT genotype $(\mathrm{MT}$ and TT) $\times$ the use of the antihypertensive drug in question. In addition, we estimated the drug-gene interaction in starters of the antihypertensive drug in question.

\section{Results \\ $\alpha$-Adducin gene (ADD1) G460W polymorphism}

Between January 1, 1990 and December 31, 1999, 6500 blood pressure measurements of 3025 hypertensive individuals, were included. In $91 \%$ of the hypertensive individuals the ADD1 genotype could be assessed. In total, 396 individuals used diuretics (559 measurements), 685 $\beta$-blockers (997 measurements), 281 calcium antagonists (366 measurements), and 294 ACE inhibitors (389 measurements). Baseline characteristics of all subjects stratified by the ADD1 genotype are presented in Table 2 . The mean DDD at baseline for users of diuretics was $0.81 \pm 0.46$, for $\beta$-blockers $0.68 \pm 0.35$, for calcium antagonists $0.77 \pm 0.34$, and for ACE inhibitors $1.11 \pm 0.65$. There was no statistically significant difference in DDDs during follow-up between the different genotypes (data not shown).

There was a significant difference in SBP levels between the genotype groups in treated subjects at the first examination. Owing to the small number of subjects with the WW genotype (7.5\% of the individuals), this group was combined with the GW genotype, group in the analysis. After adjustment for potential confounders, genotype and drug effect, the mean difference in SBP and DBP was compared between the two genotype groups for the different antihypertensive drug groups during the three examination rounds for continuous users and starters (see Figure 1). The reference group in this analysis was the mean SBP or DBP of subjects with the GG genotype of the investigated antihypertensive drug class. Diuretic users with the GW or WW genotype had a $1.25 \mathrm{mmHg}$ (95\% CI: -2.86 to 5.35 ) higher mean SBP and a $0.39 \mathrm{mmHg}$ (95\% CI: -1.95 to 2.74 ) higher mean DBP compared to diuretic users with the GG genotype.

Among starters of diuretic therapy $(N=57)$, there was no drug-gene interaction for the mean difference in SBP $(P$-value $=0.81)$ or DBP $(P$-value $=0.88)$. There was also no drug interaction among starters of $\beta$-blocker therapy $(N=63)($ SBP $P$-value $=0.95$ or DBP $P$-value $=0.75)$. However, we could only include a small number of measurements. The number starters of calcium-antagonist therapy $(N=23)$ and ACE-inhibitor therapy $(N=15)$ were too small to examine.

\section{Angiotensinogen gene (AGT) M235T polymorphism} For the AGT gene, $91 \%$ of the genotypes could be ascertained. In total, 395 individuals used a diuretic (559 measurements), 689 a $\beta$-blocker (1002 measurements), 282 a calcium antagonist (367 measurements), and 293 an ACE inhibitor (388 measurements), respectively. Baseline characteristics for all subjects stratified by AGT genotypes are presented in Table 3. There was no significant difference between the different genotype groups during the first examination. Although there was some indication that untreated subjects with the MT genotype had a higher SBP

Table 2 Baseline characteristics of all patients at the first examination stratified by $\alpha$-adducin genotype

\begin{tabular}{|c|c|c|c|c|}
\hline \multirow[b]{2}{*}{ Variable } & \multicolumn{2}{|c|}{ Untreated } & \multicolumn{2}{|c|}{ Treated } \\
\hline & $\begin{array}{c}G G \\
(N=950)\end{array}$ & $\begin{array}{c}W \text {-allele } \\
(N=602)\end{array}$ & $\begin{array}{c}G G \\
(N=469)\end{array}$ & $\begin{array}{c}W \text {-allele } \\
(N=316)\end{array}$ \\
\hline Age (years) & $69.9 \pm 8.6$ & $69.2 \pm 8.3$ & $70.7 \pm 9.1$ & $71.6 \pm 8.8$ \\
\hline SBP $(\mathrm{mmHg})$ & $153.0 \pm 21.9$ & $152.9 \pm 21.3$ & $146.0 \pm 22.7$ & $142.5 \pm 23.4^{*}$ \\
\hline DBP $(\mathrm{mmHg})$ & $78.9 \pm 12.0$ & $78.6 \pm 11.9$ & $76.0 \pm 11.8$ & $74.9 \pm 11.8$ \\
\hline \multicolumn{5}{|l|}{ Smoking } \\
\hline Current & 187 (20.0\%) & 129 (21.8\%) & 70 (15.0\%) & 59 (19.0\%) \\
\hline Past & $393(42.1 \%)$ & 259 (43.7\%) & $213(45.7 \%)$ & $134(43.2 \%)$ \\
\hline Never & 354 (37.9\%) & 205 (34.6\%) & $183(39.3 \%)$ & $117(37.7 \%)$ \\
\hline Diuretic & & & 125 (36.7\%) & $87(27.5 \%)$ \\
\hline$\beta$-Blockers & & & $203(43.3 \%)$ & 147 (46.5\%) \\
\hline Ca antagonist & & & 72 (15.4\%) & 43 (13.6\%) \\
\hline
\end{tabular}

Values are presented as means ( \pm s.d.), or number $(\%)$.

*Significantly different in treated or untreated group $(P$-value $<0.05)$. 
level compared to the other genotypes, there was no statistically significant difference in DDDs for the different genotypes.

The adjusted difference in blood pressure when treated with diuretics, $\beta$-blockers, calcium antagonists, or ACE inhibitors is presented in Figure 2. Also, for this gene no statistically significant drug-gene interaction was found.

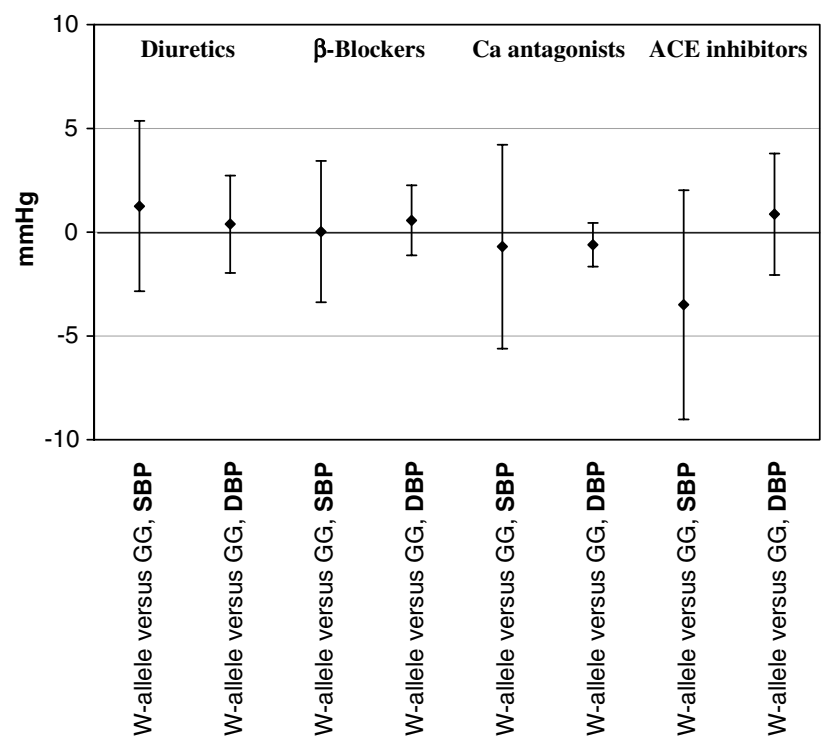

$\mathrm{SBP}=$ systolic blood pressure $\mathrm{DBP}=$ diastolic blood pressure

Figure 1 Adjusted mean difference in SBP and DBP among antihypertensive drug users with the GG genotype of the $\alpha$-adducin gene compared to subjects with the $W$-allele of $\alpha$-adducin gene. $\mathrm{SBP}=$ systolic blood pressure; $\mathrm{DBP}=$ diastolic blood pressure.
There was no drug-gene interaction for starters of diuretics (SBP $P$-value $=0.88$ or DBP $P$-value $=0.65$ ) or $\beta$ blocker therapy $($ SBP $P$-value $=0.72$ or DBP $P$-value $=0.41$ )

In addition, we assessed the effect of gene-gene interactions of the ACE I/D polymorphism, AGT M235T polymorphism, and ADD1 G460W polymorphism in treated antihypertensive drugs on the blood pressure. None of the gene-gene combinations (AGT-ADD1 or AGTACE or ADD1-ACE) modified the mean difference in SBP or DBP level.

\section{Discussion}

This study suggests that there is no interaction between the G460W polymorphism of the $\alpha$-adducin gene or the M235T polymorphism of the angiotensinogen gene and the use of diuretics, $\beta$-blockers, calcium antagonists, or ACE inhibitors (monotherapy), which resulted in different SBP or DBP levels in daily practice. Furthermore, combinations of these two polymorphisms and the I/D polymorphism of the ACE gene did not result in significant genegene-drug interactions.

The G460W and M235T polymorphisms were logical candidates to investigate as polymorphisms that could influence antihypertensive drug response. Studies with the Milan hypertensive rat and in humans with essential hypertension suggest that genetic alterations in $\alpha$-adducin may contribute to hypertension. ${ }^{15-17}$ The $\alpha$-adducin gene may affect blood pressure by increasing renal tubular reabsorption of sodium through the activation of $\mathrm{Na}^{+}, \mathrm{K}^{+}$-ATPase (adenosine triphosphatase) and the $460 \mathrm{~W}$ allele of the $\alpha$-adducin gene is associated with a higher affinity for the $\mathrm{Na}^{+}, \mathrm{K}^{+}$-ATPase pump than the

Table 3 Baseline characteristics of all patients at the first examination stratified by angiotensinogen genotype

\begin{tabular}{|c|c|c|c|c|c|c|}
\hline \multirow[b]{2}{*}{ Variable } & \multicolumn{3}{|c|}{ Untreated } & \multicolumn{3}{|c|}{ Treated } \\
\hline & $\begin{array}{c}M M \\
(N=541)\end{array}$ & $\begin{array}{c}M T \\
(N=755)\end{array}$ & $\begin{array}{c}T T \\
(N=252)\end{array}$ & $\begin{array}{c}M M \\
(N=280)\end{array}$ & $\begin{array}{c}M T \\
(N=397)\end{array}$ & $\begin{array}{c}T T \\
(N=113)\end{array}$ \\
\hline Gender (M) & $207(38.3 \%)$ & $325(43.0 \%)$ & $102(40.5 \%)$ & $111(39.6 \%)$ & $152(38.3 \%)$ & 49 (43.4\%) \\
\hline Age (years) & $69.4 \pm 8.3$ & $70.0 \pm 8.6$ & $68.8 \pm 8.4$ & $71.0 \pm 8.9$ & $70.9 \pm 8.8$ & $70.5 \pm 9.4$ \\
\hline SBP $(\mathrm{mmHg})$ & $151.3 \pm 21.9$ & $154.0 \pm 21.7$ & $152.8 \pm 21.0 *$ & $142.8 \pm 22.6$ & $145.7 \pm 23.0$ & $144.6 \pm 23.3$ \\
\hline $\mathrm{DBP}(\mathrm{mmHg})$ & $78.4 \pm 12.0$ & $79.0 \pm 12.1$ & $78.7 \pm 11.8$ & $75.3 \pm 11.6$ & $75.3 \pm 11.7$ & $77.6 \pm 12.7$ \\
\hline $\mathrm{BMI}\left(\mathrm{kg} / \mathrm{m}^{2}\right)$ & $26.4 \pm 3.6$ & $26.3 \pm 3.4$ & $26.4 \pm 3.2$ & $27.4 \pm 4.1$ & $27.0 \pm 3.6$ & $27.0 \pm 3.9$ \\
\hline Diabetes & $61(11.5 \%)$ & $90(12.3 \%)$ & $24(9.7 \%)$ & $39(13.9 \%)$ & $56(14.3 \%)$ & $16(14.4 \%)$ \\
\hline \multicolumn{7}{|l|}{ Smoking } \\
\hline Current & $100(18.9 \%)$ & $152(28.8 \%)$ & $61(24.5 \%)$ & 39 (14.1\%) & $73(18.6 \%)$ & 20 (17.9\%) \\
\hline Past & $223(42.2 \%)$ & $327(43.8 \%)$ & $101(40.6 \%)$ & $131(47.5 \%)$ & 171 (43.5\%) & 47(42.0\%) \\
\hline Never & $205(37.5 \%)$ & $267(35.8 \%)$ & $87(34.9 \%)$ & $106(38.4 \%)$ & 149 (37.9\%) & 45 (40.2\%) \\
\hline Diuretic & & & & $84(30.0 \%)$ & $106(26.7 \%)$ & 23 (20.4\%) \\
\hline$\beta$-Blockers & & & & $118(42.1 \%)$ & $185(46.6 \%)$ & 49 (43.4\%) \\
\hline Ca antagonist & & & & $39(13.9 \%)$ & $57(14.4 \%)$ & 19 (16.8\%) \\
\hline ACE inhibitor & & & & 39 (13.9\%) & 49 (12.3\%) & $22(19.5 \%)$ \\
\hline
\end{tabular}

*Significantly different in treated or untreated group $(P$-value $<0.10)$.

Values are presented as means $( \pm$ s.d.), or number (\%). 


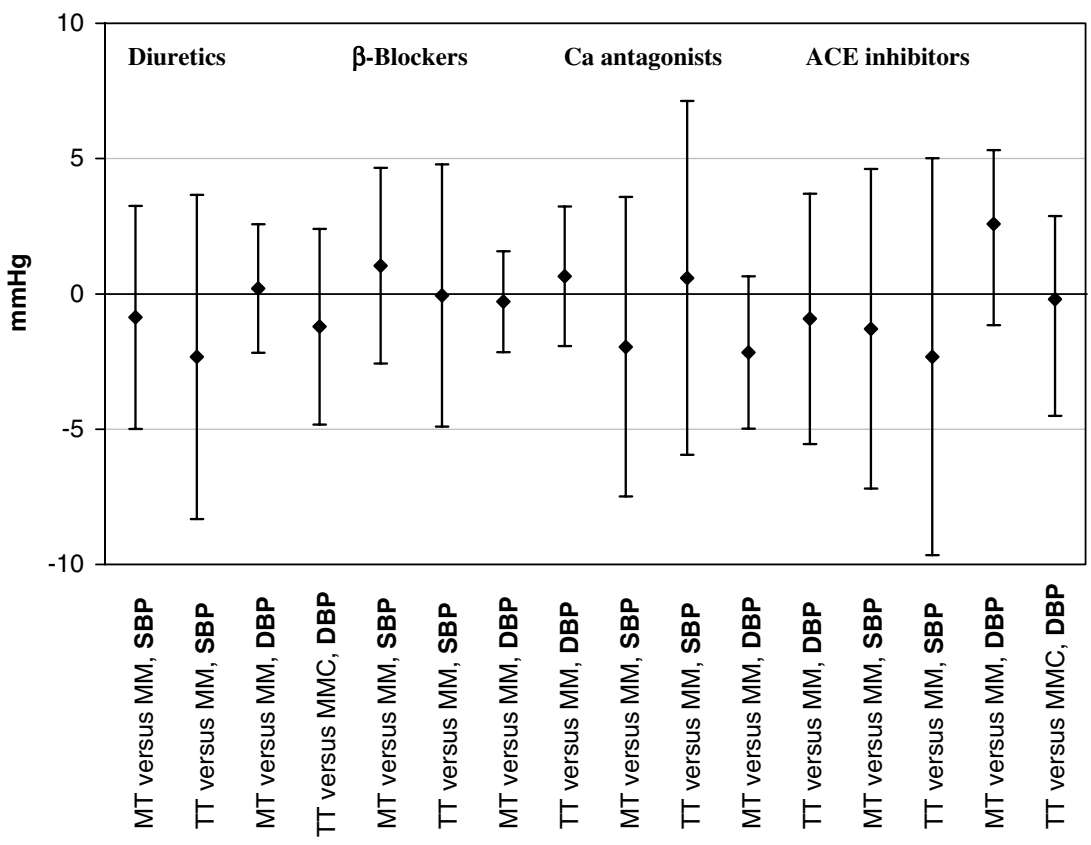

$\mathrm{SBP}=$ systolic blood pressure

$\mathrm{DBP}=$ diastolic blood pressure

Figure 2 Adjusted mean difference in SBP and DBP among antihypertensive drug users with the TT and MT genotype of the angiotensinogen gene compared to the MM genotype of the angiotensinogen gene. SBP = systolic blood pressure; $\mathrm{DBP}=$ diastolic blood pressure.

460G allele. ${ }^{18}$ Angiotensinogen is the inactive precursor of the potent vasoactive and salt-retaining hormone angiotensin II, and thus a major component of the reninangiotensin system. The M235T polymorphism of the angiotensinogen gene has an effect on plasma angiotensinogen concentration. Subjects who are homozygous $235 \mathrm{~T}$ (TT) have 15-40\% higher levels compared to subjects who are homozygous $235 \mathrm{M}(\mathrm{MM}) .{ }^{19}$ In a meta-analysis, the M235T polymorphism was significantly associated with hypertension. ${ }^{20}$ Persons with the T-allele had a higher risk of hypertension. In our study, subjects with the T-allele had a higher SBP level compared to the subjects with MM genotype at baseline. In addition, some of the trials reported an interaction between the G460W polymorphism of the $\alpha$-adducin gene or M235T polymorphism of the angiotensinogen gene on blood pressure response to antihypertensive drugs, ${ }^{1-3,7}$ although the results have been inconclusive. In daily practice, we did observe a significant difference between the genotype groups in SBP in treated groups during the first examination, but this disappeared when we analyzed the complete data.

The main difference between the previous studies and ours is that we conducted an observational study and the other studies were trials. These trials had blood pressure data prior and after the start with an antihypertensive drug. Consequently, it was possible to calculate the immediate blood pressure response. In our study, we had only measurements every 3 years. Therefore, the data were analyzed cross-sectional and consequently the mean difference in blood pressure between the genotype groups was calculated. It is possible that previously reported druggene interaction were the results of a temporary difference between the genotype groups after the start with an antihypertensive drug and therefore missed in our study. However, no drug-gene interaction was found in our group of 'starters'.

The main advantage of an observational study is that it resembles daily clinical practice. A limitation of observational studies is that they are vulnerable to confounding. For example, confounding by indication might have occurred in our study. As a physician was free to choose whether a patient receives an antihypertensive drug and which specific patients characteristics might have influenced this decision. However, the drug-gene interaction between subjects using the same antihypertensive class is most likely not influenced by this bias, since users of the same antihypertensive drug class have most likely the same characteristics and the physician is unaware of a subjects' genotype. In addition, we corrected for potential confounders, such as dose, BMI, and salt intake. Another disadvantage is that it is possible that the medication taken during the blood pressure measurement was not the initial 
drug chosen, but rather represents an alternative drug, which through a process of trail and error was found to be the most effective (good responders). However, the genotype frequencies did not differ between the 1st, 2nd, and 3rd round. Another disadvantage of our study is the overrepresentation of patients with isolated systolic hypertension. In addition, our study could not be standardized with respect to dose, agent, and duration of therapy.

Notwithstanding these caveats, this study suggests that in daily practice the G460W polymorphism of the ADD1 gene and M235T polymorphism of the AGT gene do not influence the difference in blood pressure among users of low-ceiling diuretics, $\beta$-blockers, calcium antagonist, or ACE inhibitors. Randomized clinic trials are needed to confirm our findings.

\section{Acknowledgements}

The Netherlands Heart Foundation financially supported this study, Grant number: 2001.064. The Rotterdam Study is funded by the Netherlands Organization for Scientific Research (NWO) and the Municipality of Rotterdam.

\section{References}

1 Cusi D, Barlassina C, Azzani T et al: Polymorphisms of alphaadducin and salt sensitivity in patients with essential hypertension. Lancet 1997; 349: 1353-1357.

2 Glorioso N, Manunta P, Filigheddu F et al: The role of alphaadducin polymorphism in blood pressure and sodium handling regulation may not be excluded by a negative association study. Hypertension 1999; 34 (Part 1): 649-654.

3 Sciarrone MT, Stella P, Barlassina C et al: ACE and alpha-adducin polymorphism as markers of individual response to diuretic therapy. Hypertension 2003; 41: 398-403.

4 Turner ST, Chapman AB, Schwartz GL, Boerwinkle E: Effects of endothelial nitric oxide synthase, alpha-adducin, and other candidate gene polymorphisms on blood pressure response to hydrochlorothiazide. Am J Hypertens 2003; 16: 834-839.

5 Matayoshi T, Kamide K, Takiuchi $\mathrm{S}$ et al: The thiazide-sensitive $\mathrm{Na}(+)-\mathrm{Cl}(-)$ cotransporter gene, C1784T, and adrenergic receptorbeta3 gene, T727C, may be gene polymorphisms susceptible to the antihypertensive effect of thiazide diuretics. Hypertens Res 2004; 27: 821-833.

6 Psaty BM, Smith NL, Heckbert SR et al: Diuretic therapy, the alpha-adducin gene variant, and the risk of myocardial infarction or stroke in persons with treated hypertension. JAMA 2002; 287: $1680-1689$.

7 Hingorani AD, Jia H, Stevens PA, Hopper R, Dickerson JE, Brown MJ: Renin-angiotensin system gene polymorphisms influence blood pressure and the response to angiotensin converting enzyme inhibition. J Hypertens 1995; 13 (Part 2): $1602-1609$.

8 Dudley C, Keavney B, Casadei B, Conway J, Bird R, Ratcliffe P: Prediction of patient responses to antihypertensive drugs using genetic polymorphisms: investigation of rennin-angiotensin system genes. J Hypertens 1996; 14: 259-262.

9 Kurland L, Melhus H, Karlsson J et al: Angiotensin converting enzyme gene polymorphism predicts blood pressure response to angiotensin II receptor type 1 antagonist treatment in hypertensive patients. J Hypertens 2001; 19: 1783-1787.

10 Bozec E, Fassot C, Tropeano AI et al: Angiotensinogen gene M235T polymorphism and reduction in wall thickness in response to antihypertensive treatment. Clin Sci (London) 2003; 105: 637-644.

11 Bis JC, Smith NL, Psaty BM et al: Angiotensinogen Met235Thr polymorphism, angiotensin-converting enzyme inhibitor therapy, and the risk of nonfatal stroke or myocardial infarction in hypertensive patients. Am J Hypertens 2003; 16: $1011-1017$.

12 Hofman A, Grobbee DE, de Jong PT, van den Ouweland FA: Determinants of disease and disability in the elderly: the Rotterdam Elderly Study. Eur J Epidemiol 1991; 7: 403-422.

13 Anonymous: Guidelines for ATC Classification and DDD Assignment, 3rd edn. Oslo: WHO collaberating Centre for drug statistics Methodology, 2000.

14 van Duijn CM, de Knijff P, Cruts M et al: Apolipoprotein E4 allele in a population-based study of early-onset Alzheimer's disease. Nat Genet 1994; 7: 74-78.

15 Bianchi G, Tripodi G, Casari G et al: Two point mutations within the adducin genes are involved in blood pressure variation. Proc Natl Acad Sci USA 1994; 91: 3999-4003.

16 Wang JG, Staessen JA, Barlassina C et al: Association between hypertension and variation in the alpha- and beta-adducin genes in a white population. Kidney Int 2002; 62: 2152-2159.

17 Province MA, Arnett DK, Hunt SC et al: Association between the alpha-adducin gene and hypertension in the HyperGEN Study. Am J Hypertens 2000; 13 (Part 1): 710-718.

18 Ferreri NR, Zhao Y, Takizawa H, McGiff JC: Tumor necrosis factor-alpha-angiotensin interactions and regulation of blood pressure. J Hypertens 1997; 15 (Part 1): 1481-1484.

19 Jeunemaitre X, Soubrier F, Kotelevtsev YV et al: Molecular basis of human hypertension: role of angiotensinogen. Cell 1992; 71: 169-180.

20 Sethi AA, Nordestgaard BG, Tybjaerg-Hansen A: Angiotensinogen gene polymorphism, plasma angiotensinogen, and risk of hypertension and ischemic heart disease: a meta-analysis. Arterioscler Thromb Vasc Biol 2003; 23: 1269-1275. 